\title{
INSIGHTS
}

\section{The power of human touch in the era of artificial intelligence}

\author{
Diana W. Bianchi ${ }^{1}$ \\ Pediatric Research (2019) 86:670-671; https://doi.org/10.1038/s41390-019-0484-7
}

Did you know that National Public Radio maintains a list of the best 350 commencement speeches of all time (https://apps.npr. org/commencement)? The list includes links to speeches by presidents, poets, and celebrities. I found it intimidating, until I realized that I could only identify one physician on the list, Atul Gawande. The following words are adapted from a speech I gave at the University of Maryland School of Medicine's commencement on 16 May 2019. I used the occasion to share my thoughts on the changes that have occurred in society and medical practice, education and research over the nearly four decades since I graduated from Stanford University School of Medicine. My reflections were through the lens of someone who has trained in pediatrics, neonatal intensive care, and medical genetics, which was not even a board-certified specialty when I graduated from medical school.

In my speech I highlighted three major advances in technology that have profoundly affected our lives as well as medical practice, starting with the personal computer. It is hard to imagine medicine, or even life, without the personal computer. Many of the students were even tweeting through their mobile devices as I spoke. I started receiving my first emails in 1995, around the time that many of the graduating students were born. They are a generation that has grown up with the Internet and social media as a constant companion and source of news, both individual and global. The impact of personal computers on medical care, however, was not really felt until 2009, when the federal economic stimulus package gave a financial incentive to hospitals and health-care systems to use electronic health records.

The students who were graduating from medical school on that day have never known anything but the electronic health record (EHR). While there is much to be said about the advantages of the EHR, such as its instant accessibility from any location or improved legibility of the notes, there is no doubt that the presence of the computer is a physical barrier between the patient and physician. Studies examining the impact of the EHR on the patient-physician interaction in the outpatient setting demonstrate that on average, typing into the record takes up about a third of the visit time. Other research has shown that the EHR draws the provider's attention away from the patient to the computer, impairing his or her ability to observe subtle signs of disease or discomfort. ${ }^{1}$

As an attending physician in the neonatal intensive care unit, I have personally observed that over the past 10 to 15 years, there is less and less reliance on the physical examination of the sick newborn. Sign-out rounds consist of transferring laboratory values from one covering resident to another, discussions that often occur far from the babies' bedsides. This is also a lost opportunity to directly observe physiologic changes that are made possible by the premature baby's translucent skin or to assess the entire infant as he or she lies on an open warming bed.
In medicine we have the unique privilege of touching our patients, both literally and figuratively, in the context of diagnosing and managing their illnesses. Abraham Verghese, a Professor of Medicine at Stanford University, who holds the unique title of "Vice Chair for the Theory and Practice of Medicine," has written about the sacred ritual of the physical examination, which is critical to both the establishment and maintenance of a strong physician-patient relationship. ${ }^{2}$ The role of the physical examination is increasingly threatened by more and more sophisticated diagnostic modalities, as exemplified by the primary care provider who is looking at the computer or pediatric residents doing neonatal sign out rounds. I would argue that there needs to be a return of emphasis on teaching the skills associated with active listening, the physical examination, and communication of empathy with the patient.

Dr. Wei Wei Lee, a physician-educator at the University of Chicago, has developed a curriculum to improve physician-patient interaction using the EHR. She coined the term "the first golden minute," referring to the start of the physician-patient interaction. ${ }^{3}$ She recommends that this time should be free of technology, and that the physician should make full eye contact and initiate conversation with the patient, starting with listening to the patient's concerns before typing anything into the record. Second, she recommends creating a "triangle of trust" between the patient, physician, and computer, so that both the patient and physician can simultaneously see the screen. Third, she advises sharing what is on the screen as an opportunity to discuss test results, or to use online resources as an opportunity for patient education. Since the EHR is here to stay, she believes that these approaches will continue to promote humanism in medicine.

The second major technological advance that has occurred in my practice lifetime has been the ability to sequence the human genome inexpensively and in a time frame that allows for a change in clinical management based upon the findings in the genetic code. Furthermore, DNA sequencing is available to the general population through widely advertised Internet sites. It is estimated that over 26 million people have participated in health or ancestry testing using their salivary DNA (https://www. technologyreview.com/s/612880/more-than-26-million-peoplehave-taken-an-at-home-ancestry-test/). This has profoundly changed the concepts of family and ethnic identity. Does your family consist of the people who raised you? Or does your family consist of the people with whom you share segments of the DNA code?

In medical genetics, a specialty in which we call ourselves the "Sherlock Holmes of medicine," we typically perform an extremely thorough physical examination looking for subtle clues missed by other health-care providers, such as unusual hair patterns or preauricular tags. One of the most interesting consults I have ever received was from a pediatric nurse who called me from the

${ }^{1}$ Eunice Kennedy Shriver National Institute of Child Health and Human Development, National Institutes of Health, Bethesda, MD, USA

Correspondence: Diana W. Bianchi (Diana.Bianchi@nih.gov)

Received: 4 June 2019 Accepted: 14 June 2019

Published online: 25 June 2019 
inpatient ward about a one-year-old who was admitted for an infection. Her question: Is it normal for a one-year-old to have silvery grey hair? This case was memorable because it was the only time in my professional career in which the consult team and I literally pulled out our hair, as well as the patient's, to make the diagnosis. Under the microscope we observed the differences between the patient's hair, which lacked melanosomes, and our own. This led to the diagnosis of Griscelli syndrome, a rare autosomal recessive disorder that is associated with immune system dysfunction and a predisposition to lethal infections. ${ }^{4}$ Establishing the correct diagnosis led to the infant's lifesaving treatment, which was a bone marrow transplant. Perhaps at some point in the future, when all newborns will receive their DNA sequence along with their birth certificate, it will be known from the start that an infant like this one has mutations in both copies of her RAB27A gene. What I found remarkable about the case was that until she was hospitalized, no one apparently noticed her abnormal hair color, or if they did, they did not think to question whether or not it was clinically significant.

Much to the dismay of departmental budget administrators, medical geneticists also pride themselves on the amount of time they spend talking to the patient about his or her family history, again looking for clues that have been overlooked by others. For example, such a conversation may reveal that the parents of the child are first cousins, which might also suggest the presence of an autosomal recessive disorder. Yet even in medical genetics, there is now an emphasis on performing genome sequencing to quickly end the diagnostic odyssey of patients who have mysterious disorders. Furthermore, in a specialty in which pattern recognition is critical for the diagnosis of a dysmorphic child, there is an increasing use of software programs that use facial photographs to suggest diagnoses. ${ }^{5}$ While these technical advances are wonderful, they should be employed in addition to a comprehensive physical examination.

This brings me to the third advance, artificial intelligence (Al) and machine learning, in which computers are trained with enormous data sets to recognize differences between healthy and diseased tissue. While many of the initial Al studies developing and applying machine-learning classifiers have already shown impressive results for imaging applications in radiology and pathology, analysis of EHRs has proven to be more challenging. A recent study from China, however, described the design of a data mining framework for EHR data that employed a deep learning natural language processing system to extract clinical information from free text data. ${ }^{6}$ Records from over 1.3 million pediatric outpatient visits were used to develop a hierarchical diagnostic framework based on organ systems and 55 common pediatric conditions, such as asthma, bronchiolitis, sepsis, and diarrhea. The
Al model's diagnostic performance was better than junior physician groups, but slightly worse than senior physicians.

My remarks focused on three technologies that have transformed both medicine and society during the past four decades: the personal computer, DNA sequencing, and artificial intelligence. How will these technologies affect the future practices of the graduating students? Furthermore, what will be the (now) unimaginable technical advances that will occur as their careers evolve? Over the next four decades, the technologies that they will use to do their job-and live their lives-will certainly change. One thing that will not change, however, is the power of observation and the healing properties of human touch. We need both to provide optimal care.

While it was natural for the graduating students to feel apprehension and anxiety about what lay ahead as they crossed the threshold from student to doctor, they could already take comfort in the fact that they will help patients when they look them directly in the eye and listen to them. I advised them that they should think of the healing power of human touch as a technology that will never become outdated and as something that they will always carry with them wherever they go.

\section{ACKNOWLEDGEMENTS}

The author would like to thank Drs. Shari Barkin and Constantine Stratakis for helpful discussions regarding this overall topic.

\section{ADDITIONAL INFORMATION}

Competing interests: The author declares no competing interests.

Publisher's note: Springer Nature remains neutral with regard to jurisdictional claims in published maps and institutional affiliations.

\section{REFERENCES}

1. Hedian, H. F., Greene, J. A. \& Niessen, T. M. The electronic health record and the clinical examination. Med Clin. N. Am. 102, 475-483 (2018).

2. Costanzo, C. \& Verghese, A. The physical examination as ritual. Med Clin. N. Am. 102, 425-431 (2018)

3. Alkureishi, M. A., Lee, W. W., Webb, S. \& Arora, V. Integrating patient-centered electronic health record communication training into resident onboarding: curriculum development and post-implementation survey among house staff. JMIR Med Educ. 4, e1 (2018).

4. Ménasché, G. et al. Mutations in RAB27A cause Griscelli syndrome associated with haemophagocytic syndrome. Nat. Genet. 25, 173-176 (2000).

5. Gurovich, Y. et al. Identifying facial phenotypes of genetic disorders using deep learning. Nat. Med. 25, 60-64 (2019).

6. Liang, $\mathrm{H}$. et al. Evaluation and accurate diagnoses of pediatric diseases using artificial intelligence. Nat. Med. 25, 433-438 (2019). 